\title{
Nanopillars of light
}

Illuminating cells through tiny transparent pillars permits spatially confined excitation of fluorescent molecules.

Put a materials scientist and a cellular biophysicist in a room together, and what do you get? There are many possible outcomes, and as reported in a recent paper, one of them is light delivered to the inside of cells with tiny nanopillars.

Bianxiao Cui and Yi Cui, both at Stanford University and only coincidentally with the same name, were college classmates in China some years ago. When they both wound up with faculty positions in different departments at Stanford University, they decided to collaborate. Yi Cui, a materials scientist, was working on nanostructures for batteries and solar cells, and Bianxiao Cui set out to find a way to apply this to her work on imaging single molecules in neurons.

"One of the biggest problems in singlemolecule imaging is the fluorescence background," says Bianxiao Cui, and the researchers thought that nanotechnology could help. Existing methods - total internal reflection fluorescence (TIRF) microscopy, for instance —use an evanescent wave to generate a spatially confined zone of excitation so that only a small fraction of molecules are excited and the fluorescence background is reduced. TIRF, however, has the problem of only restricting the light in one dimension. What the researchers wanted was a way to restrict light to a small volume in three dimensions.

An approach that uses what are called zero-mode waveguides does achieve this. In this approach, very tiny holes are drilled into a layer of metal coated onto glass, holes so small that instead of propagating light that is shone onto the metal, they restrict the light within the hole. "Our technique is in some ways similar to the zero mode waveguides," says Bianxiao Cui, "but instead of making a hole [in the metal] we have a pillar that is poking out." The researchers constructed transparent nanopillars 100-150 nanometers in diameter on the metal-coated substrate; these are small enough, again, that

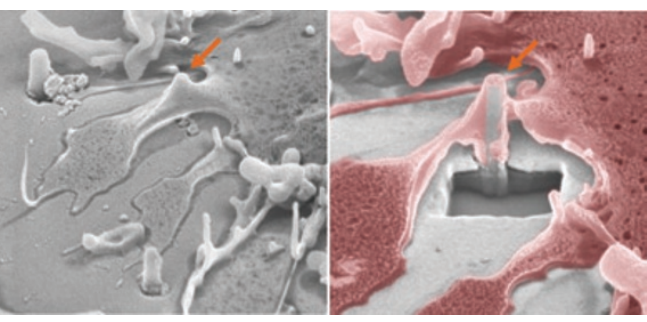

Scanning electron micrographs of a cell on nanopillars. Image courtesy of Bianxiao Cui.

light shone onto the substrate mostly does not pass through it. A certain amount of light does propagate through the pillar, however, and generates a three-dimensional evanescent wave around its surface.

"The best thing," says Bianxiao Cui, "is that this interfaces with cells very nicely. The cells love it." The result, then, is the possibility for spatially very confined optical access to the interior of the cell. Illumination through nanopillars of a cell that expresses GFP cytosolically, for instance, gives very confined spots of fluorescence only at the pillar locations.

\section{SIGNAL TRANSDUCTION}

\section{DISSECTING SIGNALING AT THE SPEED OF LIGHT}

\section{Researchers use light-activated photo-uncaging to dissect the elementary steps in protein kinase signaling networks in living human cells.}

Protein kinase signaling pathways are complicated processes, which involve elementary steps that operate at different rates. To quantitatively understand kinase signaling pathways, the network connectivity and the kinetics of the individual steps must be thoroughly mapped.

Network connectivity can be assessed by knocking out a protein kinase using genetic methods or small interfering RNA, but these techniques do not allow one to study the impact on signaling pathways in real time. Further, these techniques do not allow the signaling pathway function of a kinase to be isolated from its other potential functions in a cell. Ideally, what one really needs is a method to rapidly turn on the catalytic function of a specific kinase in a living cell, without perturbing the kinase's other functions.

Jason Chin of the Medical Research Council Laboratory of Molecular Biology in Cambridge, UK and his colleagues now report a powerful approach to dissect the elementary steps in the Raf-MEK-ERK signaling pathway that is central to cell proliferation. They had previously designed a photocaged version of the amino acid lysine, which could be site-specifically incorporated into proteins in cells in response to a reassigned stop codon via an evolved pyrrolysyl-tRNA synthetase-tRNA pair. They now have adapted the method to create a version of the kinase MEK1 that can be activated in living human cells with light.

ATP is necessary for kinase phosphorylation activity. Protein kinases contain a highly conserved lysine residue in the ATPbinding pocket of the catalytic domain. By simply replacing this lysine with the bulkier photocaged version, Chin and his colleagues blocked the binding of ATP. Then, when they shone a light on the cell, the caging group was photochemically cleaved, leaving behind a native lysine residue that allows ATP binding to occur. To ensure that MEK1 would be immediately active once the caging group was removed, they substituted two serine residues with aspartic acid to mimic the phosphorylated state.

The method allows them to "activate signaling from any point in the pathway, where we cage a particular kinase," explains Chin. "That's a much more powerful tool than stimulating the top of the pathway and watching the output at the bottom without the resolution of what's happening at the intermediate steps." In the Raf-MEK-ERK pathway, Raf phosphorylates MEK1, activating it; MEK1 in turn phosphorylates ERK1 and ERK2. ERK1 and ERK2 have two phosphorylation sites each; both sites must be phosphorylated 
The researchers used simulations to model the decay of the light at the tip and the sides of the nanopillars (the light emerges mostly from the sides). They estimate the observation volume to be $10^{-16}$ litres, an order of magnitude smaller than that achieved with twophoton excitation. They have grown cortical neurons, hippocampal neurons, cardiomyocytes and a variety of mammalian cell lines on these pillars, and in all cases the cells seem to thrive. "Because of the small dimensions of the pillar, the cell seems to treat it as some sort of cellular organelle, to recognize those dimensions," Bianxiao Cui says. The researchers continue to investigate the best dimensions and interpillar distances for various applications.

Although others have in the past used nanopillars to deliver molecules to cells, the data at this stage are not entirely conclusive about whether the pillars are topologically inside or outside the cell (that is, whether they access the cytosol or not). Scanning electron micrographs show that the cells seem to engulf the pillars, but the resolution is insufficient to tell whether or not there is a membrane between the pillar and cytoplasm. This will affect the types of experiments that can be done: for instance, recruiting cytosolic molecules to the pillars will only be possible if they are actually inside the cell.

Irrespective of the topology, the pillars can still be used to optically excite molecules in a very small volume. Bianxiao Cui and her lab members are pursuing experiments in which they use the nanopillars to either locally photo-uncage a neurotransmitter or to excite a small number of photoactivatable fluorescent proteins so that the behavior of single molecules can then be studied as they diffuse to dark areas in the cell.

\section{Natalie de Souza}

\section{RESEARCH PAPERS}

Xie, C. et al. Vertical nanopillars for highly localized fluorescence imaging. Proc. Natl. Acad. Sci. USA 108, 3894-3899 (2011).

before the proteins will translocate into the nucleus to activate transcription factors. Although stimulation of the whole network has been found to result in a lag phase before ERK accumulation in the nucleus, the elementary kinetic steps of this process had not been previously resolved. By photo-uncaging the primed MEK1 and then using time-lapse microscopy to follow the fate of EGFP-tagged ERK in the cells, the researchers confirmed that the diphosphorylation step determined the rate of the appearance of ERK in the nucleus.

When they stimulated the whole network, they observed that ERK accumulated in the nucleus, reaching a peak, but then rapidly dissipated. They did not see this with the photocaged MEK1, which is only regulated by light and not by Raf. This suggests that a negative feedback mechanism is at play.

The method could be especially powerful for determining the kinetics of each of the elementary steps in a signaling pathway. This could be achieved by photocaging each of the kinases in the pathway in turn, which should be relatively straightforward to do because of the conserved lysine in the ATP-binding pocket. "By comparing the kinetics when you activate at different points in the pathway," says Chin, "you'll be able to say something about the rate of potentially every step in the pathway and make quantitative models."

\section{Allison Doerr}

\section{RESEARCH PAPERS}

Gautier, A. et al. Light-activated kinases enable temporal dissection of signaling networks in living cells. J. Am. Chem. Soc. 133, 2124-2127 (2011).

\section{NEWS IN BRIEF}

\section{NEUROSCIENC}

\section{Dissecting neural networks with Brainbow}

Neurotropic viruses — which replicate in neurons and can cross synapses - are useful as neural circuit tracing tools. Card et al. combine this technology with the Brainbow approach to highlight neuronal connections within a larger network. They inserted the Brainbow cassette into the genome of an engineered pseudorabies virus, which resulted in the expression of either yellow or cyan fluorescent reporters in response to Cre recombinase-mediated recombination, allowing synaptic connections to be traced.

Card, J.P. et al. Proc. Natl. Acad. Sci. USA 108, 3377-3382 (2011).

IMAGING

\section{A label for electron cryotomography}

Electron cryotomography allows cells, organelles and macromolecular assemblies to be imaged at high resolution in a near-native state. A major challenge, however, is the identification of cellular structures of interest. Wang et al. describe the use of a ferritin protein as a clonable fusion label for electron cryotomography. Ferritin consists of a protein shell with a central cavity that can be loaded with iron, providing an electron-dense tag for image contrast.

Wang, Q. et al. Structure 19, 147-154 (2011).

\section{GENOMICS}

\section{A population-scale variation map}

Mills et al. applied different methods, including paired-end mapping, depth-of-coverage analysis, split-read mapping and sequence assembly, to detect structural variants on a population scale. The resulting variant map encompasses deletions, insertions and tandem duplications, more than $50 \%$ of which could be mapped at base-pair resolution. This resource will be valuable for the understanding of the origin of variants, their genotyping in large cohorts and for disease-association studies.

Mills, R.E. et al. Nature 470, 59-65 (2011).

\section{SENSORS AND PROBES}

\section{Oligodeoxyfluorosides for imaging}

Guo et al. present oligodeoxyfluoroside (ODF) fluorophores, made up of a DNA backbone in which fluorophores replace the DNA bases; energy transfer between the fluorophores allows a wide variety of emission colors to be generated with a single excitation wavelength. The authors conjugated the ODF fluorophores to antibodies and showed that they could be used for multicolor cellular imaging.

Guo, J. et al. Proc. Natl. Acad. Sci. USA 108, 3493-3498 (2011).

\section{NEUROSCIENCE}

\section{Optogenetics toolkit for primates}

With optogenetics, light is harnessed to control specific neurons in the brain. Beyond basic neuroscience research applications, optogenetics has the potential to treat brain injury and disease. Diester et al. now present a set of optogenetics tools for experiments in nonhuman primates. They characterized the safety and efficiency of opsin expression in the rhesus monkey cortex. They also describe a fiber-optic device for making minimally invasive, repeated in vivo fluorescence measurements over time. Diester, I. et al. Nat. Neurosci. 14, 387-397 (2011). 